\title{
Shorter drug testing intervals are associated with improved drug misuse rates
}

Jeff Gudin, MD; Neel Mehta, MD; F. Leland McClure, PhD; Justin K. Niles, MA; Harvey W. Kaufman, MD

\section{ARTICLE INFO}

Keywords:

repeat drug testing

follow-up time intervals

opioids

nonprescribed drug use

prescription drug compliance

DOI:10.5055/jom.2020.0591

(C) 2020 Journal of Opioid Management, All Rights Reserved.

\section{ABSTRACT}

Objective: The Centers for Disease Control and Prevention (CDC) recommend that clinicians prescribing opioids for chronic pain should consider at least annual urine drug testing (UDT). We evaluated whether shorter intervals for repeat UDT are associated with decreased rates of drug misuse.

Design: Retrospective analysis of deidentified serial UDT and matched prescribing data.

Setting: We analyzed Quest Diagnostics 2016-2017 UDT results from new patients being monitored for prescription drug adberence, in nonsubstance use disorder (SUD) treatment environments.

Main Outcome Measures: Drug misuse was defined as the absence of a prescribed substance or the presence of a nonprescribed substance. Patients with $\geq 3$ sets of the UDT results were included.

Results: UDT results from 49,601 patients (148,803 specimens) were tested. Declines in misuse between the first and second UDT were bighest for those tested at the shortest intervals: approximately weekly, 19 percent; monthly, 15 percent; bimonthly, 12 percent; quarterly, 9 percent; semiannually, 3 percent; misuse rates increased by 1 percent for patients tested annually. Declines in misuse were more pronounced for opioids than other drug groups. Substantial declines in positivity were noted for heroin (32 percent) and nonprescribed fentanyl (10 percent). Declines in misuse between the second and third UDT followed a similar pattern.

Conclusions: UDT intervals of $\leq$ quarterly were associated with marked declines, but testing annually or semiannually was not associated with consistent decreases. Our findings suggest that clinical strategies that include serial testing conducted quarterly or sooner may be instrumental in decreasing drug misuse. Testing more frequently than "at least once annually" should be considered by clinicians monitoring potential drug misuse.

\section{INTRODUCTION}

Overdose deaths in the United States are increasing at an alarming rate. Between 2014 and 2017, these deaths increased by at nearly 50 percent, from 47,055 to $70,237 . .^{1}$ In 2019 , this represented one in every 39 deaths. Heroin-related overdose deaths rose from 12,989 in 2015 to 15,482 in 2017 (a 19 percent increase), while overdose deaths related to synthetic opioids other than methadone (eg, illegally manufactured fentanyl) increased from 9,580 to 28,466 (197 percent). ${ }^{1}$ Over 35 percent of opioid overdose deaths in 2017 involved a prescription opioid. ${ }^{2}$ A recent publication suggested that states may be greatly underestimating the effect of opioid-related overdose deaths because of incomplete cause-of-death reporting, indicating that the opioid overdose epidemic may be worse than it appears. ${ }^{3}$

The 2016 Centers for Disease Control and Prevention (CDC) Guideline for Prescribing Opioids 
for Chronic Pain includes 12 practice recommendations to improve opioid risk-benefit communications between clinicians and patients, improve safety and effectiveness of pain treatment, and reduce risks associated with long-term opioid therapy, including opioid use disorder and overdose. The guideline recommendation for assessing risk and addressing harms states that, "clinicians should use urine drug testing before starting opioid therapy and consider urine drug testing at least annually to assess for prescribed medications as well as other controlled prescription drugs and illicit drugs." Although urinebased drug testing is recognized as a vital clinical tool for safe prescribing of controlled substances, ${ }^{4-9}$ there is no established standard for recommended time intervals between repeat drug tests. This may be due to the paucity of studies and data concerning the utility of treatment strategies including repeat urine drug tests (UDTs) to reduce drug misuse.

The limited literature does, however, support that repeat drug testing is associated with reductions in nonprescribed/illicit drug use. ${ }^{10-13}$ One study showed that a control group of "high-risk" patients with repeat drug testing had virtually identical misuse rates to those of "low-risk" patients. ${ }^{14}$ Unfortunately, most of these studies were based on a relatively small number of patients, and to the best of our knowledge, the association of time intervals between follow-up UDTs and drug misuse rates has never been explored. The purpose of this study was to analyze a large national reference laboratory database to examine how rates of overall drug misuse, nonprescribed or illicit drug positivity, and compliance with prescribed drugs change for different time intervals between repeat UDTs.

\section{METHODS}

\section{Study data}

All prescription drug monitoring test results from the Quest Diagnostics medMATCH ${ }^{\circledR}$ reporting service from 2016 through 2017, which included a company-wide unique patient identification number to enable patient tracking over time, were selected for potential inclusion. medMATCH patient reports correlate a patient's prescribed medication with results from testing for the presence or absence of prescribed and nonprescribed $\operatorname{drug}(\mathrm{s})$ and drug metabolite(s). Prescription drug data are provided by the clinicians ordering the tests, who are instructed to include information for all prescribed drugs tested for the patient, not just the drugs tested they have prescribed. To compile a complete list of medications, clinicians rely upon patient self-report, state prescription monitoring programs, and other electronic databases.

We required that each patient's first UDT have data for at least one drug prescribed by the ordering clinician, limiting our analysis to patients fitting our definition of being monitored for prescription drug compliance $(n=823,515)$. Patients in specific substance use disorder (SUD) treatment settings (identified by providers) were excluded, to make our results more applicable to the general patient population being monitored for drug compliance in the United States (leaving 763,476 specimens). We then excluded any patients with a test result in the 9 months preceding the study, from March 2015 through December 2015, to define and enable analysis of "new" patients (leaving 573,895 specimens). Finally, we required each patient to have at least three separate specimens tested during the study period (of which we analyzed results from the first three), leaving a final analytical set of 148,803 specimens from 49,601 patients (Figure 1). We chose three specimens in order to assess whether patterns of changing misuse observed between UDT1 and UDT2 were observed again between UDT2 and UDT3.

\section{Drug use definitions}

Positivity for nonprescribed drugs was defined as the presence of a positive result for any drugs not listed as prescribed by the ordering clinician, or for recreational/illicit drugs. Noncompliance with prescribed drugs was defined as a negative result for a drug listed as prescribed by the ordering clinician. "Drug misuse" was defined as either nonprescribed positivity or noncompliance (or both) occurring on a UDT. We analyzed changes in the proportion of patients who demonstrated nonprescribed use and, separately, changes in the number positive results for nonprescribed drugs and mirrored these results for noncompliance with prescribed drugs. We believe that analysis of changes in the number of drugs could be considered more comprehensive as patients can decrease their nonprescribed use (for example, from 2 drugs to 1 drug), but not be counted in the proportion of patients who no longer demonstrate nonprescribed use. Throughout the text we use the term 


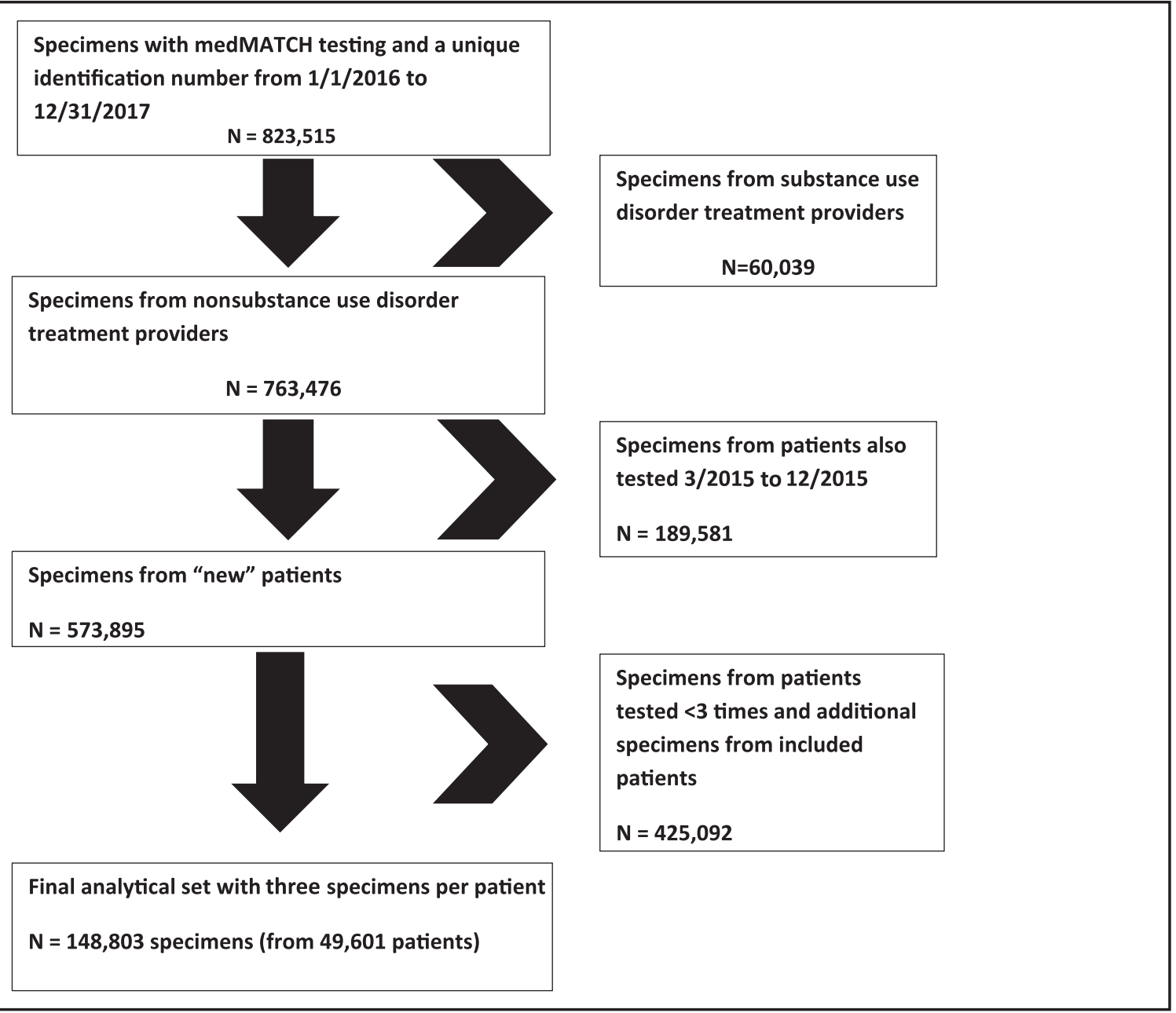

Figure 1. Exclusion flow diagram.

"decline" to describe when aggregate drug misuse rates for the entire study population, specific demographic subgroups, or misuse of specific drug groups, were lower at UDT2 than at UDT1, and/or lower at UDT3 than at UDT2.

\section{Specimen collections and handling}

Clinical laboratory analysis of all UDT results included either presumptive immunoassay screening tests confirmed by quantitative definitive mass spectrometry, or tests performed directly by quantitative definitive mass spectrometry. Presumptive immunoassay screening tests with low cross-reactivity (eg, 6-aminoclonazepam, lorazepam, hydromorphone) were performed using test procedures modified to detect the compounds. Liquid chromatography-tandem mass spectrometry tests were performed to provide the definitive quantitative analysis of the drugs and drug metabolites. Quantitative confirmation analysis was performed to identify the drugs and drug metabolites detected and to rule out false-positive presumptive screening results. All mass-spectrometry methods were validated using National Institute of Standards and Technology (NIST) traceable reference materials.

\section{Drug classes}

For analysis of drug classes, each class includes the parent $\operatorname{drug}(\mathrm{s})$ and drug metabolite(s). The "amphetamines" class includes both amphetamine and methamphetamine, but not MDMA (which is a separate class). The "opiates" class includes codeine, morphine, hydrocodone, and hydromorphone. The "oxycodone" class includes oxycodone and oxymorphone. A class was counted positive if any of the parent drugs or metabolites were present. Not all patients 
were tested for all classes, because perceived medical necessity for certain tests varies among physicians ordering the tests. We also analyzed data by three meta-groups: "opioids," "nonopioid medications," and "other drugs." We did not report drug classes with less than 30 inconsistencies in specimens from the initial time frame (UDT1) in the tables, owing to small numbers, but these classes were included in our aggregate analyses of misuse. The classes not shown for each table are listed in the footnotes.

\section{Demographic factors}

This study included patient results from 47 states. Provider specialty type was provided to Quest Diagnostics by the clinician. Metropolitan types were based on the 2013 Rural-Urban Continuum Codes obtained from the United States Department of Agriculture Economic Research Service. ${ }^{15}$ Federal Information Processing Standard (FIPS) codes from the USDA were connected to patient ZIP Codes ${ }^{\circledR}$ from their UDT via SAS version 9.4 (SAS Institute, Cary, North Carolina) internal maps dataset. We grouped data into three categories: "large metro" (counties in metropolitan areas of 1 million population or more), "small metro" (counties in metropolitan areas of less than 1 million population), and "nonmetro" (counties not in metropolitan areas).

\section{Time intervals}

The time intervals are a combination of the intervals between a patient's first specimen (UDT1) and their second specimen (UDT2), as well as the intervals between UDT2 and the patient's third specimen (UDT3). Testing 1-3 weeks apart was defined as "weekly." We then used 2 weeks before and after each 30 day interval to define "monthly," "bimonthly," and "quarterly" testing. This resulted in the following intervals: weekly, <22 days between tests; monthly, 22-45 days; bimonthly, 46-74 days; quarterly, 75-104 days; semiannual, 105-330 days; annual, >330 days. These groups were chosen due to common physician-ordering patterns. Patients can be tested at different intervals between UDT1-2 and UDT2-3.

\section{Statistical analyses}

Statistical significance testing of group proportions was conducted using the Chi-square test. Trends in proportions of misuse among various groups and proportion of patients tested at various time intervals were analyzed using the Cochran-Armitage test. The analysis of drug meta-groups was based on the summation of all drug classes within the meta-group. Data analyses were performed using SAS ${ }^{\circledR}$ Studio 3.6 on $S_{A S}{ }^{\circledR} 9.4$ (SAS Institute, Inc., Cary, North Carolina). This Quest Diagnostics Health Trends ${ }^{\mathrm{TM}}$ study was deemed exempt by the Western Institutional Review Board (Puyallup, Washington).

\section{RESULTS}

Between UDT1 and UDT2, rates of drug misuse declined for patients tested semiannually or sooner: by 18.8 percent for weekly testing, 14.5 percent monthly, 12.4 percent bimonthly, 9.0 percent quarterly, and 2.7 percent semiannually. Patients tested only annually demonstrated a 1.2 percent increase in drug misuse (Figure 2). At UDT1, drug misuse rates were 16.9 percent higher in patients tested weekly than in those tested annually (66.1 percent versus 49.2 percent), but only 3.9 percent higher at UDT2 (53.7 percent versus 49.8 percent).

Between UDT2 and UDT3, drug misuse rates declined for patients tested quarterly or sooner: 10.6 percent for weekly testing, 9.2 percent monthly, 6.9 percent bimonthly, and 2.7 percent quarterly. Patients tested at semiannual and annual intervals demonstrated a 3.5 percent and 0.5 percent increase in drug misuse, respectively (Figure 2). At UDT2, drug misuse rates were 10.9 percent higher in patients tested weekly between UDT2 and UDT3 than in those tested annually (56.9 percent versus 46.0 percent), but only 4.7 percent higher at UDT3 (50.9 percent versus 46.2 percent).

Table 1 examines drug misuse rates for various demographic factors on UDT1 and UDT2 by the time intervals between these tests. Overall, rates of drug misuse declined from 57.7 percent on UDT1 to 52.1 percent on UDT2. There was a decline in drug misuse from UDT1 to UDT2 for every demographic factor studied. Nearly, every demographic factor studied also showed a significant trend in misuse decline for the shorter time interval groups. Most factors also demonstrated a significant trend in drug misuse at UDT1, with the highest proportion of misusers being tested at the shortest intervals.

Table 2 examines drug misuse rates for various demographic factors on UDT2 and UDT3 by the time intervals between these tests. Overall, rates of drug misuse declined from 52.1 percent on UDT2 to 


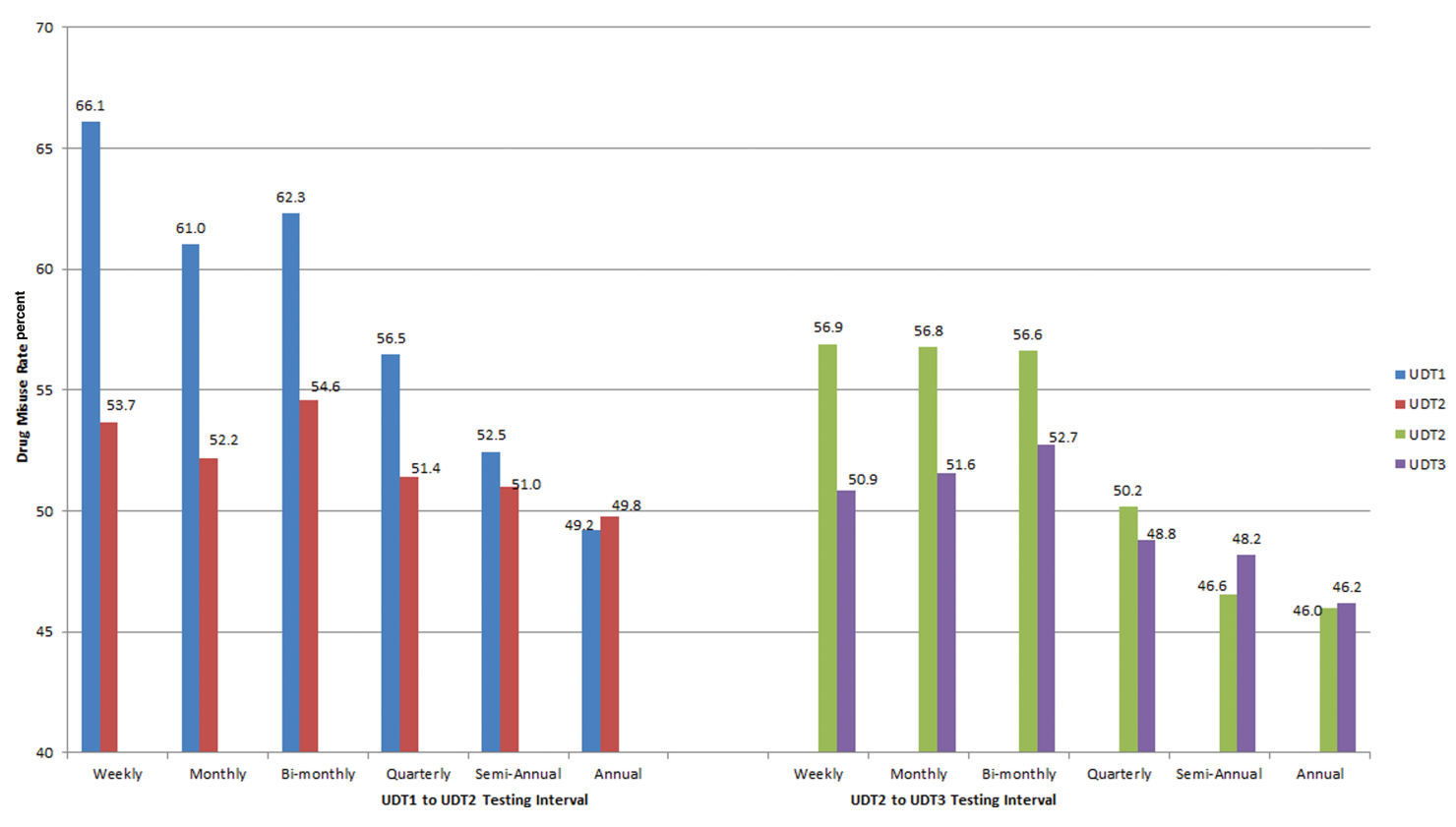

Figure 2. Drug misuse rates from UDT1 to UDT2 and from UDT2 to UDT3, by testing interval; UDT-urine drug test. Rates shown for UDT2 above differ because patients can be tested at different time intervals from UDT1-UDT2 and UDT2-UDT3.

50.0 percent on UDT3. There was a decline in drug misuse from UDT2 to UDT3 for every demographic factor studied, except in patients $<25$ years of age. Again, nearly every factor studied showed a significant trend in misuse decline for the shorter interval groups. Most factors demonstrated a significant trend in drug misuse at UDT2, with the highest proportion of misusers being tested at the shortest intervals.

Patients with shorter intervals between UDT1 and UDT2 had higher rates of nonprescribed drug positivity on UDT1 (weekly patients: 52.2 percent; annual patients 30.7 percent) ( $p<0.01$ for trend). They were also more likely to test positive for multiple nonprescribed drugs $(\mathrm{p}<0.01$ for trend, Figure 3). Patients testing positive for heroin were significantly more likely to be tested at shorter intervals (56.7 percent weekly versus 12.9 percent semiannual and annual combined, $\mathrm{p}<0.01)$. The same was true for cocaine ( 40.8 percent versus 15.0 percent, $\mathrm{p}<0.01$ ) and nonprescribed fentanyl ( 44.8 percent versus 17.7 percent, $\mathrm{p}<0.01$ )

Overall, the number of positive results for nonprescribed drugs declined between UDT1 and UDT2 (Figure 4). The extent of decline was significantly associated with UDT frequency: weekly, 21.0 percent; monthly, 15.7 percent; bimonthly, 11.2 percent; quarterly, 4.8 percent; and semiannual, 0.2 percent
( $\mathrm{p}<0.01$ for trend). However, for patients tested annually, the number of positive results for nonprescribed drugs increased by 6.7 percent between UDT1 and UDT2. The extent of decline in the proportion of patients testing positive for nonprescribed drugs was significantly associated with UDT time interval as well: 16.0 percent weekly (from 52.2 percent to 43.8 percent), 12.4 percent monthly (41.6-36.5 percent), 9.7 percent bimonthly (39.435.5 percent), and 5.9 percent quarterly (37.1-34.9 percent). Increases were demonstrated for patients tested semiannually ( 0.5 percent, from 34.6 percent to 34.8 percent) and annually (3.5 percent, from 30.7 percent to 31.8 percent). A similar trend was noted for declines in the number of nonprescribed positive test results between UDT2 and UDT $3(\mathrm{p}<$ 0.01 ) and in the proportion of patients testing positive for nonprescribed drugs: 10.0 percent weekly (from 44.8 percent to 40.3 percent), 7.8 percent monthly (39.2-36.1 percent), 8.2 percent bimonthly (37.1-34.0 percent), 4.3 percent quarterly (34.4-32.9 percent), and 2.0 percent annually (31.4-30.8 percent). An increase was demonstrated for patients tested semiannually (3.4 percent, from 31.8 percent to 32.9 percent).

Shorter intervals between drug tests were associated with larger declines in the number of 


\begin{tabular}{|c|c|c|c|c|c|c|c|c|c|c|c|c|c|c|c|c|c|c|c|c|c|c|c|c|c|}
\hline \multirow{8}{*}{ 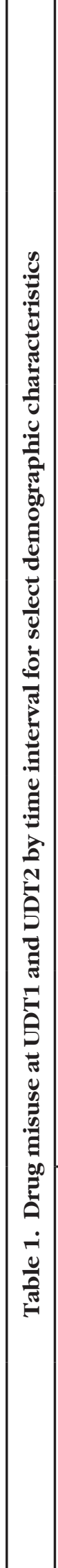 } & & 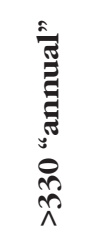 & & 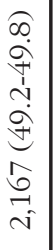 & & 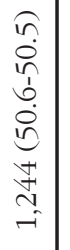 & 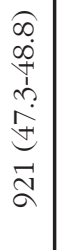 & & 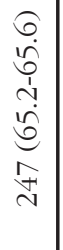 & 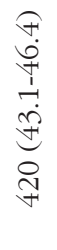 & 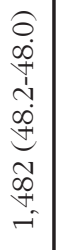 & & 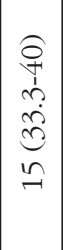 & 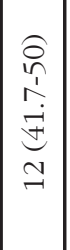 & $\begin{array}{l}\hat{n} \\
0 \\
n \\
\tilde{n} \\
\dot{1} \\
n \\
n \\
n \\
n\end{array}$ & $\begin{array}{l}\hat{n} \\
\hat{n} \\
\hat{n} \\
i n \\
\hat{n} \\
\hat{n} \\
0\end{array}$ & 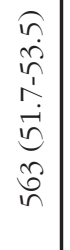 & 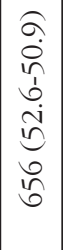 & 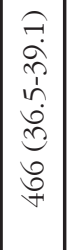 & & 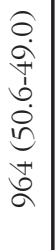 & 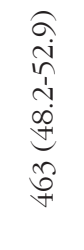 & 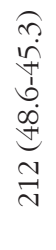 & $\begin{array}{l}\vec{r} \\
i n \\
\stackrel{1}{r} \\
i n \\
n \\
r\end{array}$ & 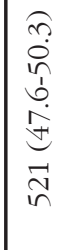 \\
\hline & & 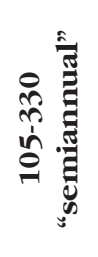 & & 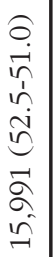 & & $\begin{array}{l}\tilde{n} \\
\vec{n} \\
b \\
i \\
i \\
\infty \\
\infty \\
\infty \\
\approx \\
\approx\end{array}$ & 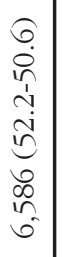 & & $\begin{array}{l}6 \\
\stackrel{0}{0} \\
0 \\
\vdots \\
\hat{0} \\
0 \\
m \\
\infty \\
- \\
-1\end{array}$ & 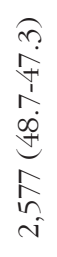 & 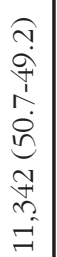 & & 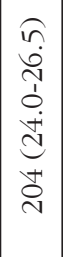 & 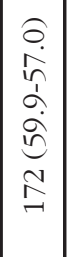 & 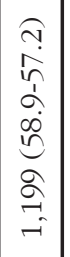 & 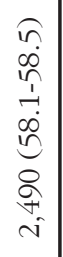 & 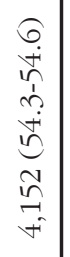 & 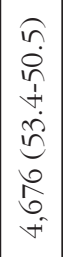 & 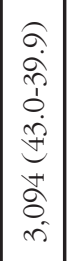 & & 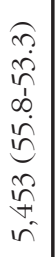 & 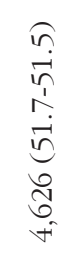 & 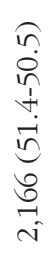 & 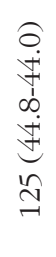 & 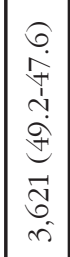 \\
\hline & 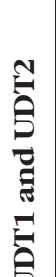 & 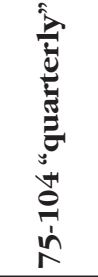 & & 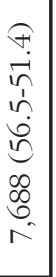 & & 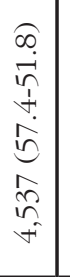 & $\begin{array}{l}\hat{\sigma} \\
\hat{\infty} \\
\hat{n} \\
\hat{n} \\
\hat{n} \\
\hat{n} \\
\tilde{n}\end{array}$ & & 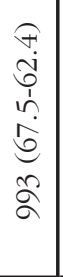 & 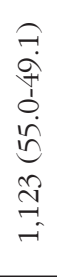 & 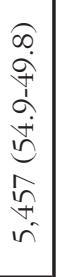 & & 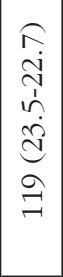 & 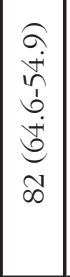 & 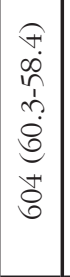 & 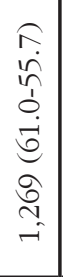 & 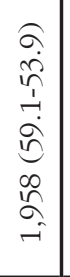 & $\begin{array}{l}\infty \\
0 \\
0 \\
0 \\
0 \\
0 \\
0 \\
0 \\
\vec{N} \\
\sim \\
i \\
i\end{array}$ & 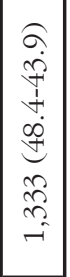 & & 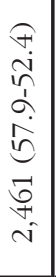 & 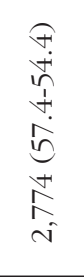 & 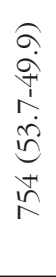 & $\begin{array}{l}\widehat{\partial} \\
\underset{j}{+} \\
\stackrel{+}{+} \\
\vec{d} \\
\vec{\sigma} \\
\vec{a}\end{array}$ & $\begin{array}{l}0 \\
0 \\
0 \\
+1 \\
1 \\
10 \\
i n \\
\infty \\
0 \\
0 \\
-1\end{array}$ \\
\hline & 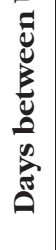 & 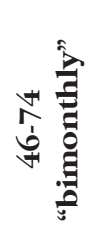 & & 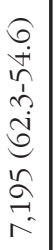 & & 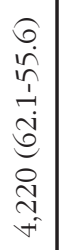 & 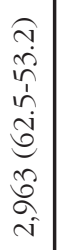 & & 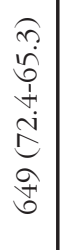 & 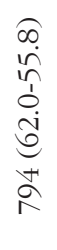 & $\begin{array}{l}\hat{n} \\
\tilde{n} \\
\hat{n} \\
\overrightarrow{0} \\
0 \\
o \\
0 \\
i \\
i n\end{array}$ & & 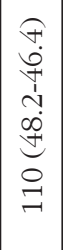 & 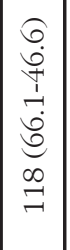 & 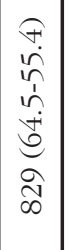 & 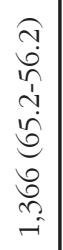 & 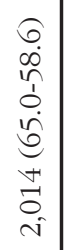 & 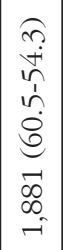 & 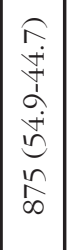 & & 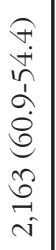 & 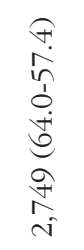 & $\begin{array}{l}\hat{n} \\
i n \\
\hat{n} \\
\hat{n} \\
\text { } \\
\infty \\
\infty \\
i n \\
i n\end{array}$ & 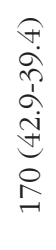 & 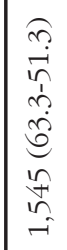 \\
\hline & & 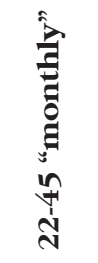 & & 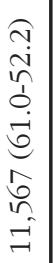 & & 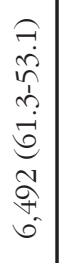 & $\begin{array}{l}\hat{\sigma} \\
0 \\
n \\
b \\
\dot{\delta} \\
0 \\
n \\
\hat{o} \\
i n \\
i n\end{array}$ & & 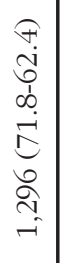 & 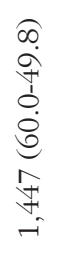 & 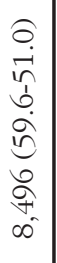 & & 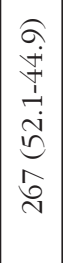 & 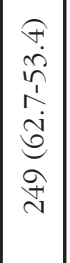 & 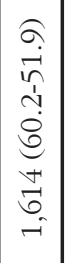 & 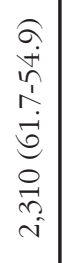 & 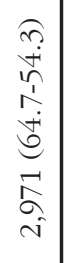 & 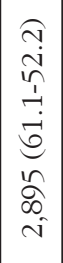 & 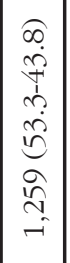 & & 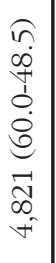 & 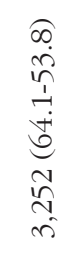 & 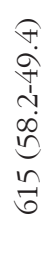 & 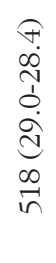 & 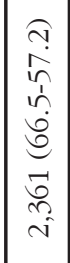 \\
\hline & & 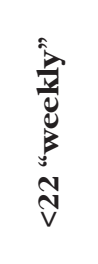 & & 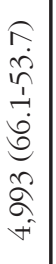 & & 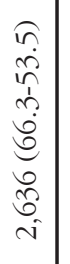 & $\begin{array}{l}\hat{\alpha} \\
\hat{n} \\
\hat{\alpha} \\
\hat{\omega} \\
\hat{e} \\
\hat{v} \\
\hat{N} \\
i\end{array}$ & & 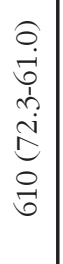 & $\begin{array}{l}0 \\
\dot{0} \\
\hat{n} \\
0 \\
0 \\
\infty \\
0 \\
\hat{0} \\
\dot{\infty} \\
\dot{+}\end{array}$ & 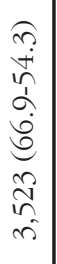 & & $\begin{array}{l}\sigma \\
\infty \\
\infty \\
\infty \\
\infty \\
i \\
\hat{\sigma} \\
o \\
\infty\end{array}$ & 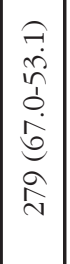 & 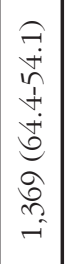 & 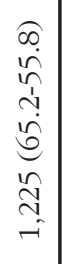 & $\begin{array}{c}r \\
\hat{n} \\
i n \\
i \\
\hat{n} \\
\hat{\sigma} \\
0 \\
0 \\
0 \\
- \\
-\end{array}$ & 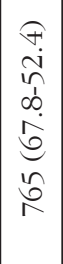 & 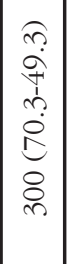 & & $\begin{array}{c}0 \\
0 \\
i \\
n \\
0 \\
0 \\
0 \\
0 \\
0 \\
0 \\
n \\
v \\
v \\
v\end{array}$ & 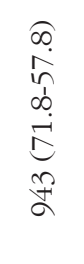 & 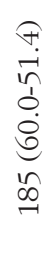 & 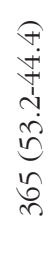 & 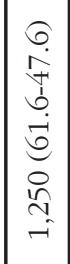 \\
\hline & & 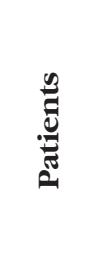 & 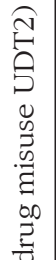 & 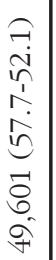 & & 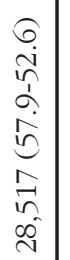 & 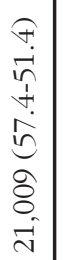 & & 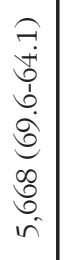 & 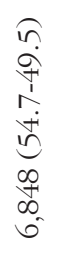 & 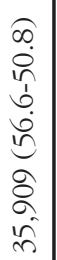 & & 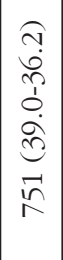 & 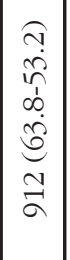 & 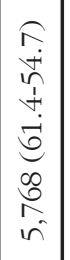 & 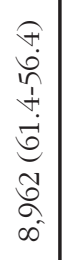 & 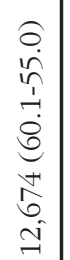 & 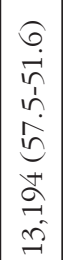 & 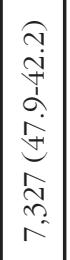 & & 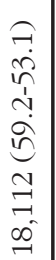 & 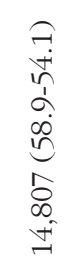 & $\begin{array}{l}6 \\
i \\
i n \\
+ \\
+\dot{0} \\
i 0 \\
8 \\
i n \\
\forall+\end{array}$ & 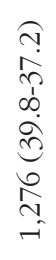 & 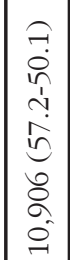 \\
\hline & & & 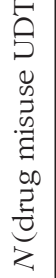 & 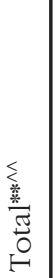 & $\begin{array}{l}\dot{\vec{v}} \\
\overrightarrow{\tilde{D}} \\
\overrightarrow{0}\end{array}$ & 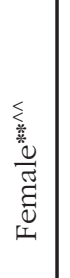 & 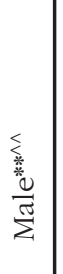 & 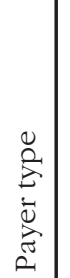 & 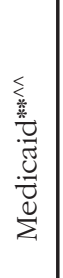 & 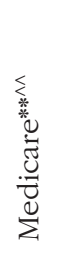 & 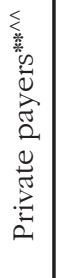 & $\begin{array}{l}0 \\
0 \\
5 \\
5 \\
8 \\
4 \\
4\end{array}$ & 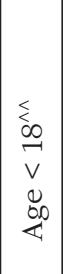 & $\begin{array}{l}n \\
v \\
v \\
v \\
\Delta \\
\dot{s} \\
v i \\
v 1 \\
\infty \\
\sim\end{array}$ & 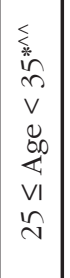 & 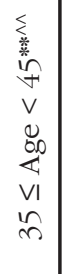 & 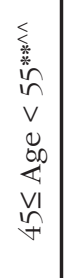 & 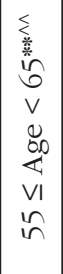 & 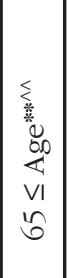 & 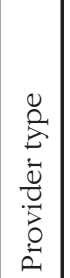 & 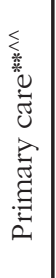 & 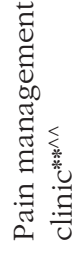 & 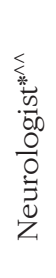 & 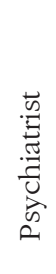 & 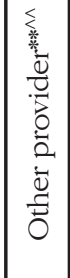 \\
\hline
\end{tabular}


nonprescribed drugs found for opioids, nonopioid medications, and other drugs between UDT1 and UDT2 ( $<0.01$ for trend, Table 3). There was an overall decline in the number of nonprescribed drugs found for most drug groups between UDT1 and UDT2. There was a significantly larger decline in the number of nonprescribed opioids (15.3 percent) than there was for nonopioid medications (6.4 percent, $\mathrm{p}<0.01$ ) and other drugs (8.0 percent, $\mathrm{p}<0.01)$. There was a larger decline in the number of nonprescribed positive tests for heroin (32.2 percent) than any other drug group. Between UDT2 and UDT3, nonprescribed drug use declined for all drug groups except heroin, amphetamines, and cocaine. Nonprescribed opioid use declined at a greater rate (9.0 percent) than nonprescribed use of nonopioid medication ( 4.1 percent, $\mathrm{p}<0.01$ ) and other drugs (1.2 percent, $\mathrm{p}<0.01)$. Except for tramadol, nonprescribed use of opioid subgroups declined with weekly, monthly, bimonthly, and quarterly testing (Table 4).

Between UDT1 and UDT2, the extent of decline in the proportions of patients demonstrating noncompliance with their prescribed drugs was significantly associated with UDT time interval as well: 31.5 percent weekly (from 29.6 percent to 20.3 percent), 21.6 percent monthly (34.9-27.4 percent), 16.1 percent bimonthly (37.7-31.6 percent), 15.3 percent quarterly (32.0-27.1 percent), semiannually (7.6 percent, 29.4-27.1 percent), and annually (2.8 percent, from 29.3 percent to 28.5 percent) (Figure 5). The declines were stronger for opioid medications on the whole than for nonopioid medications, but every drug group studied except methylphenidate and tricycic antidepressants exhibited a decline in noncompliance (Table 5). Noncompliance with prescribed drugs declined between UDT2 and UDT3. The extent of the decline was significantly associated with UDT time interval as well: 15.0 percent weekly (from 23.7 percent to 20.1 percent), 13.4 percent monthly (31.1-26.9 percent), 6.5 percent bimonthly (32.5-30.4 percent), and 1.5 percent quarterly (25.9-25.5 percent). Increases were demonstrated for patients tested semiannually ( 4.2 percent, from 24.2 percent to 25.2 percent) and annually (2.1 percent, from 24.0 percent to 24.5 percent) (Figure 5). Between UDT2 and UDT3, noncompliance with prescribed opioids declined by 9.4 percent but increased slightly for nonopioid medications ( 0.5 percent). Patients tested quarterly or sooner demonstrated declines in opioids, while only the groups 


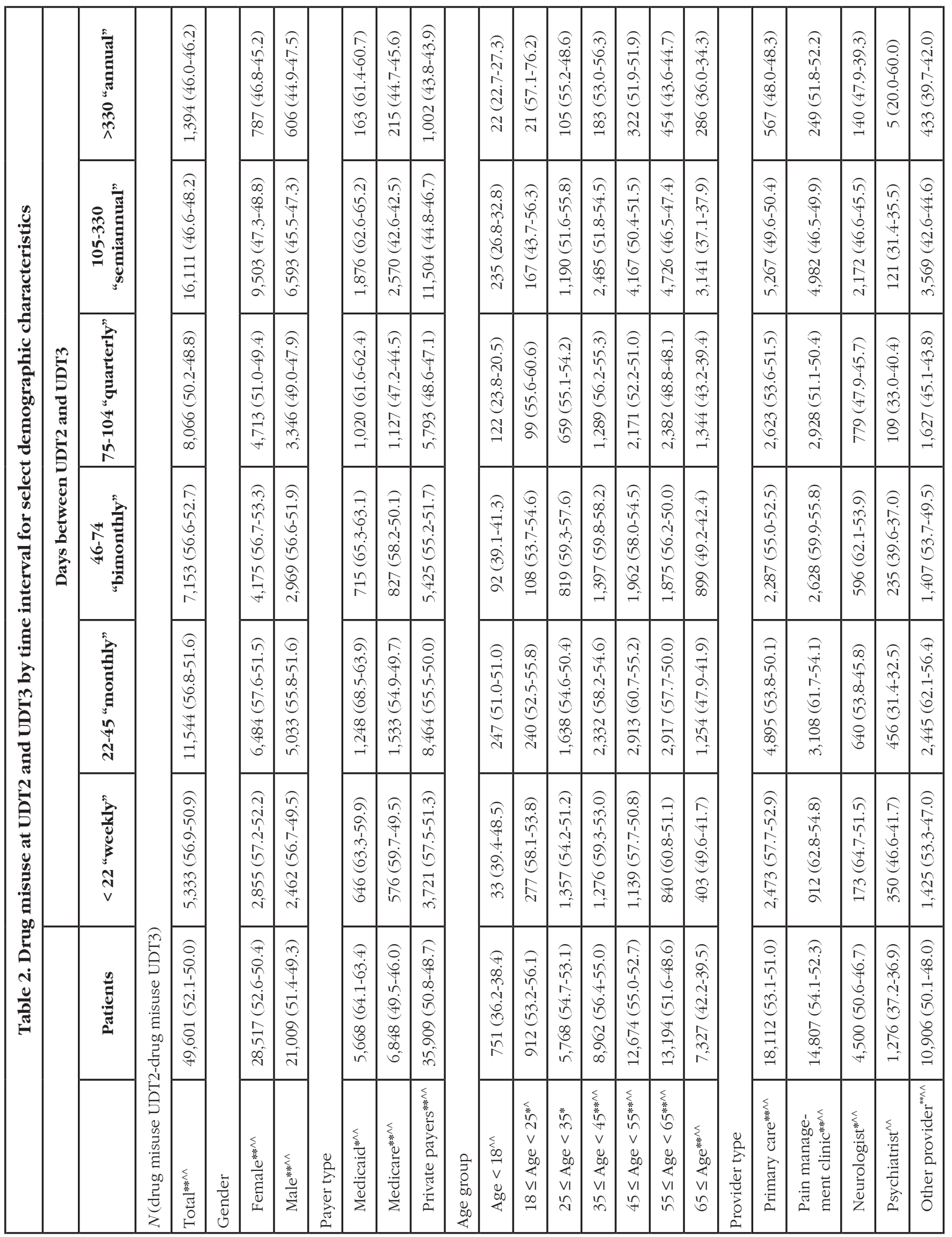


tested monthly or sooner demonstrated declines for nonopioid medications (Table 6).

\section{DISCUSSION}

The findings of the present study demonstrate that UDT testing intervals of quarterly or less were associated with aggregate reductions in both prescription drug misuse and illicit drug use. Less frequent testing was not consistently associated with decreases in misuse over time. The data showed an overall decline in drug misuse from UDT1 to UDT3 for every demographic factor studied. Most individual demographic factors studied showed a significant trend in drug misuse decline for the shorter time interval groups from UDT1-2 and again from UDT2-3. We observed declines in positivity for nonprescribed drugs and declines in noncompliance with prescribed drugs. For both of these types of potentially dangerous misuse, the declines were strongest for opioids. Semiannual and annual testing was not associated with consistent declines in misuse for their respective populations; however, this study showed that shorter-interval repeat UDT was associated with meaningful declines in aggregate drug misuse, especially as related to opioids.

Previous work has shown that high-risk patients who receive repeat drug testing have drug misuse outcomes very similar to those of low-risk patients in specific populations. ${ }^{14}$ The data presented in this study appear to confirm these assertions in a much larger population. Our study suggests that prescribing clinicians are good assessors of patient risk and are testing the highest risk patients at the shortest time intervals. Patients tested at weekly intervals had the highest rate of misuse on UDT1 (66.1 percent), while those tested annually had the lowest (although still substantial) rate of misuse on UDT1 (49.2 percent). Patients tested weekly had the highest prevalence of positivity for multiple nonprescribed drugs (22.5 percent) and were disproportionately testing positive for the most dangerous and addictive drugs (heroin, cocaine, and nonprescribed fentanyl). They also tended to be younger than patients tested at longer intervals. Yet, despite all of these factors, results from the first follow-up UDT showed much more similar misuse rates from the same patients (53.7 percent weekly versus 49.8 percent annually).

It is worth noting that even patients determined to be at the lowest risk, as evidenced by the testing 


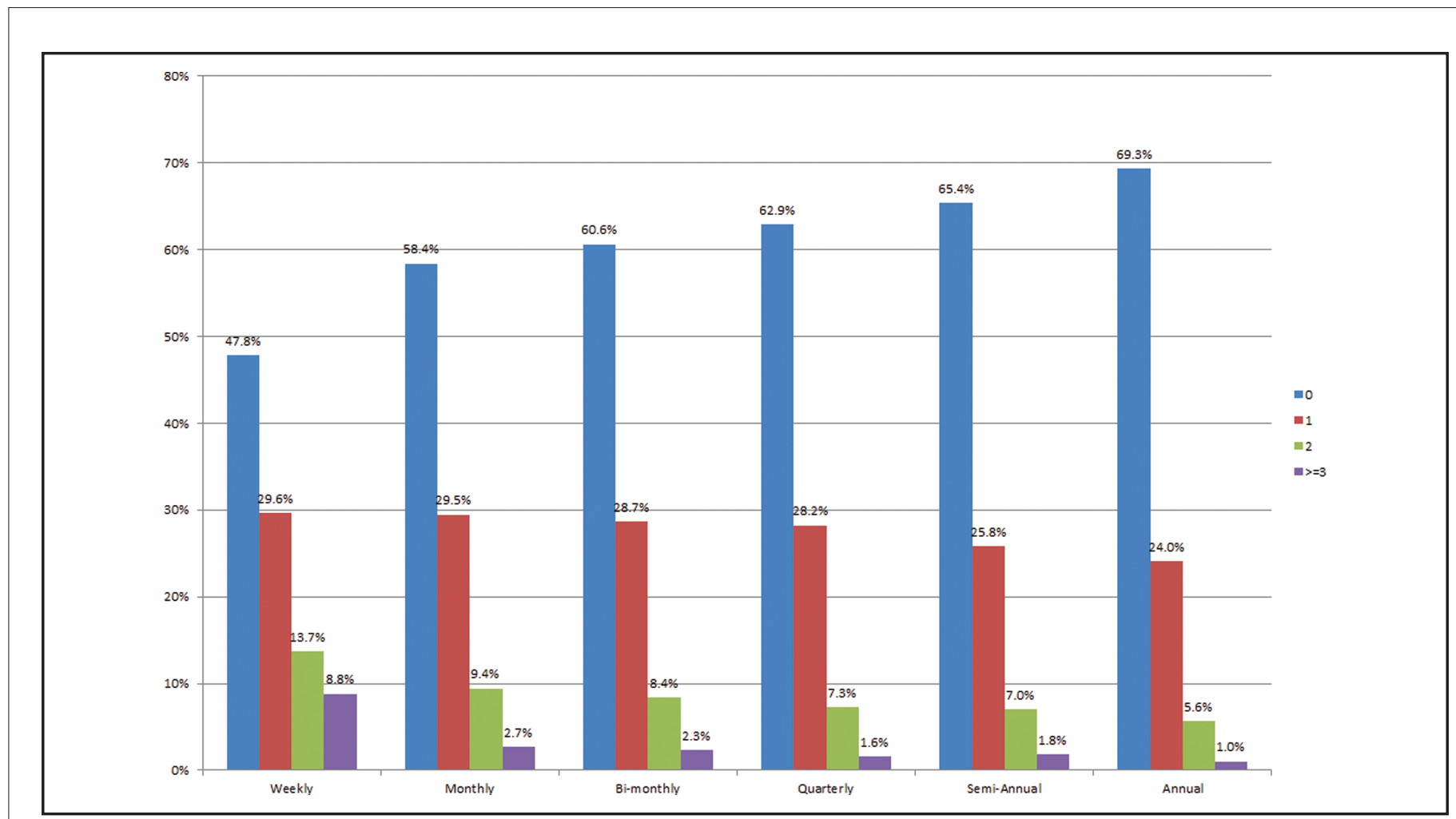

Figure 3. Number of positive results for nonprescribed drugs on UDT1 by testing interval; UDT-urine drug test.

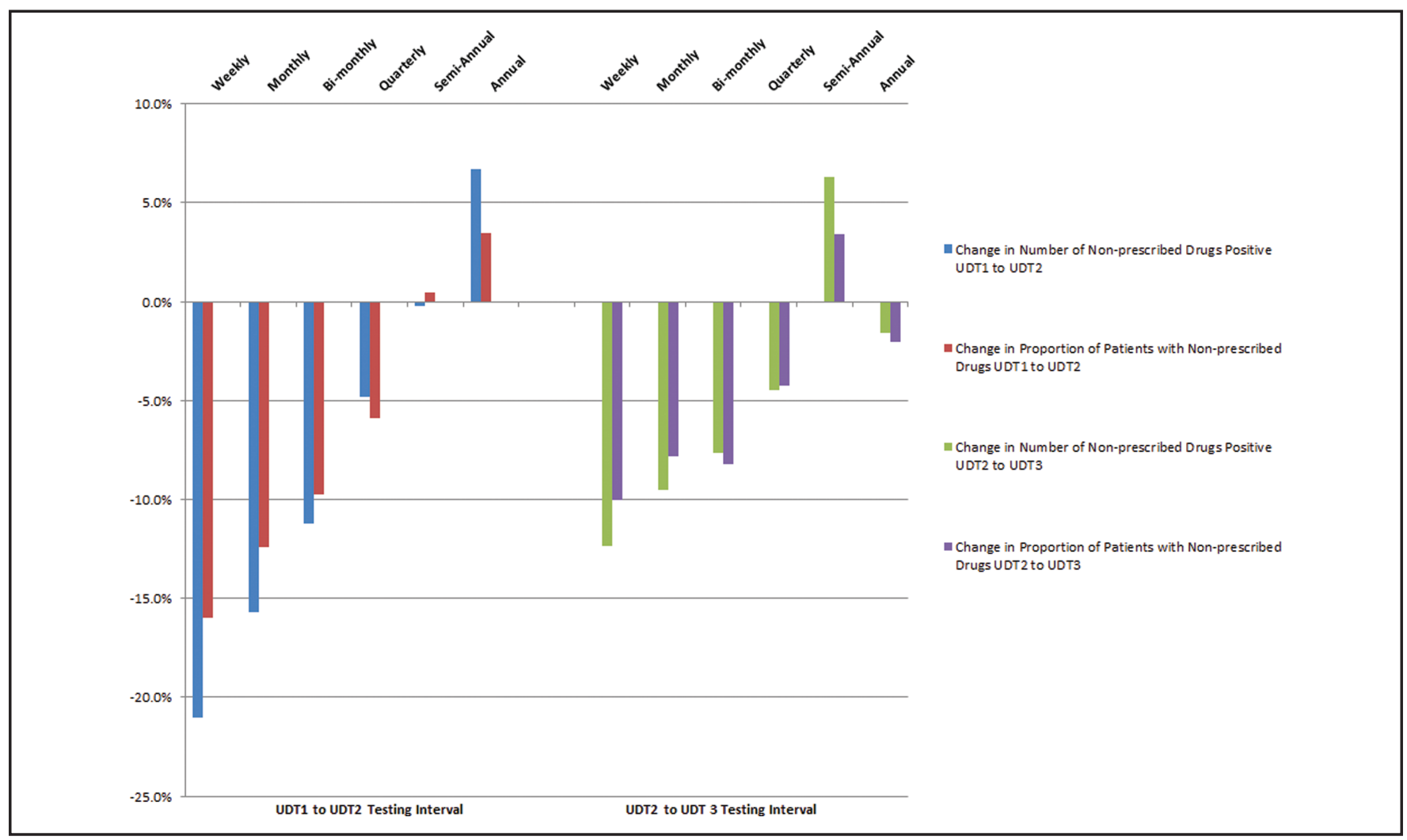

Figure 4. Change in number of nonprescribed drugs and proportion of patients testing positive for nonprescribed drugs from UDT1 to UDT2 and from UDT2 to UDT3, by testing interval. UDT-urine drug test. Patients can be tested at different time intervals from UDT1-UDT2 and UDT2-UDT3. 
This document is licensed under Creative Commons CC-BY-NC-ND-4.0 for non-commerical use from 12/16/2020 thru 12/16/2023. All Rights Reserved. Commerical use requires additional licensing. Please visit www.copyright.com for additional licensing options.

Table 3. Change in number of positive test results for nonprescribed drug groups from UDT1 to UDT2, by testing interval

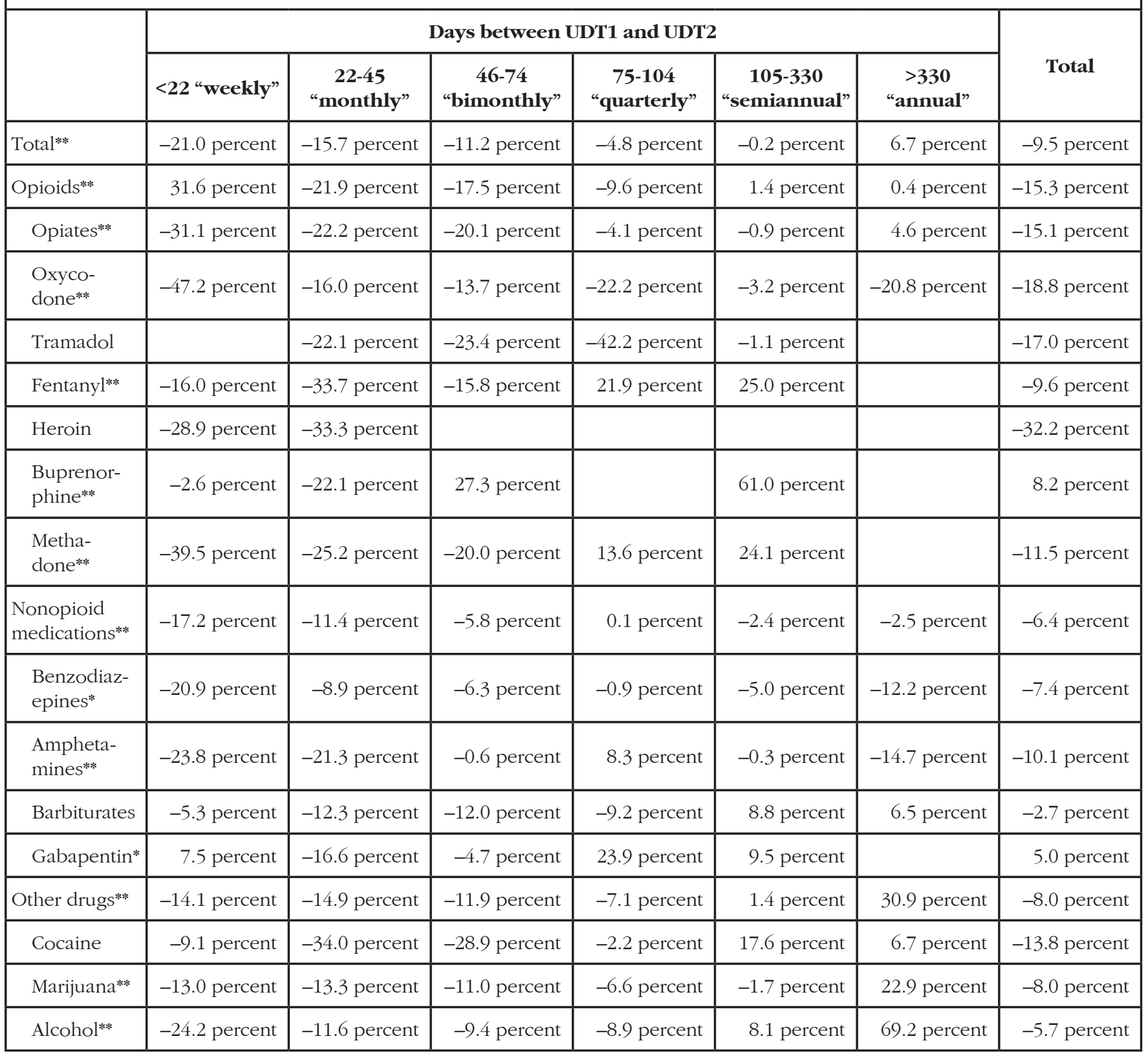

interval chosen by the prescribing physician, still had misuse rates near 50 percent. These findings raise two considerations: first, patients initially considered lower risk might have also benefitted from treatment strategies including shorter intervals between UDTs; second, that while our data suggest that meaningful aggregate declines in misuse can be achieved through treatment strategies that include frequent repeat drug testing in presumably higherrisk groups, overall misuse remains high even in the presumably lowest risk groups. UDT as advocated by the CDC and other opioid prescribing guidelines is just one component of the overall strategy of assessing risk and addressing harms of opioid use.

A consensus statement adopted by the American Society of Addiction Medicine (ASAM) Board of Directors and endorsed by the American College of Medical Toxicology provided guidance about the effective use of drug testing in the identification, diagnosis, treatment, and promotion of recovery for patients with, or at risk for, addiction. This statement notes that there is no magic formula for determining 
This document is licensed under Creative Commons CC-BY-NC-ND-4.0 for non-commerical use from 12/16/2020 thru 12/16/2023. All Rights Reserved. Commerical use requires additional licensing. Please visit www.copyright.com for additional licensing options.

Table 4. Change in number of positive test results for nonprescribed drug groups from UDT2 to UDT3, by testing interval

\begin{tabular}{|c|c|c|c|c|c|c|c|}
\hline & \multicolumn{6}{|c|}{ Days between UDT2 and UDT3 } & \multirow[b]{2}{*}{ Total } \\
\hline & <22 "weekly" & $\begin{array}{c}22-45 \\
\text { "monthly" }\end{array}$ & $\begin{array}{c}46-74 \\
\text { "bimonthly" }\end{array}$ & $\begin{array}{c}\text { 75-104 } \\
\text { "quarterly" }\end{array}$ & $\begin{array}{c}105-330 \\
\text { "semiannual" }\end{array}$ & $\begin{array}{c}\text { >330 } \\
\text { "annual" }\end{array}$ & \\
\hline Total** & -12.4 percent & -9.5 percent & -7.7 percent & -4.5 percent & 6.3 percent & -1.6 percent & -4.5 percent \\
\hline Opioids** & -21.6 percent & -17.8 percent & -8.8 percent & -8.6 percent & 9.9 percent & -6.8 percent & -9.0 percent \\
\hline Opiates ${ }^{* *}$ & -22.4 percent & -17.5 percent & -13.5 percent & -6.7 percent & 6.8 percent & 1.2 percent & -9.8 percent \\
\hline $\begin{array}{l}\text { Oxyco- } \\
\text { done** }\end{array}$ & -21.3 percent & -20.3 percent & -6.1 percent & -11.4 percent & 5.6 percent & -23.4 percent & -10.0 percent \\
\hline Tramadol & 15.4 percent & -30.8 percent & 19.5 percent & -12.1 percent & 27.5 percent & -60.0 percent & -0.8 percent \\
\hline Fentanyl* & -20.9 percent & -11.7 percent & -4.7 percent & -6.1 percent & 31.5 percent & -28.6 percent & -8.5 percent \\
\hline Heroin & -9.3 percent & & & & & & 4.3 percent \\
\hline $\begin{array}{l}\text { Buprenor- } \\
\text { phine* }\end{array}$ & -33.3 percent & -11.8 percent & -19.6 percent & -14.7 percent & 43.3 percent & & -10.5 percent \\
\hline Methadone* & -31.0 percent & -13.9 percent & -12.3 percent & -17.5 percent & 13.9 percent & 100.0 percent & -10.4 percent \\
\hline $\begin{array}{l}\text { Nonopioid } \\
\text { medications }\end{array}$ & -7.1 percent & -3.9 percent & -8.7 percent & -5.3 percent & 0.0 percent & -8.5 percent & -4.1 percent \\
\hline $\begin{array}{l}\text { Benzodiaz- } \\
\text { epines }\end{array}$ & -8.7 percent & -6.3 percent & -7.4 percent & -7.2 percent & -5.1 percent & -11.0 percent & -6.6 percent \\
\hline $\begin{array}{l}\text { Ampheta- } \\
\text { mines** }\end{array}$ & 1.8 percent & 0.0 percent & 2.9 percent & 21.0 percent & 37.6 percent & 7.7 percent & 12.3 percent \\
\hline Barbiturates & -16.3 percent & 5.3 percent & -17.6 percent & -10.3 percent & -2.0 percent & -20.0 percent & -6.0 percent \\
\hline Gabapentin & -13.3 percent & 0.7 percent & -27.4 percent & -17.7 percent & 8.8 percent & 0.0 percent & -6.9 percent \\
\hline Other drugs** & -9.1 percent & -8.4 percent & -5.4 percent & -0.2 percent & 13.0 percent & 11.9 percent & -1.2 percent \\
\hline Cocaine ${ }^{* *}$ & -11.9 percent & -9.6 percent & 29.9 percent & 43.9 percent & 73.4 percent & 87.5 percent & 7.4 percent \\
\hline Marijuana** & -5.7 percent & -7.0 percent & -5.4 percent & -2.4 percent & 7.3 percent & 5.8 percent & -1.9 percent \\
\hline Alcohol** $^{* *}$ & -18.5 percent & -12.0 percent & -12.9 percent & -0.3 percent & 18.9 percent & 18.4 percent & -2.1 percent \\
\hline
\end{tabular}

NOTE: The metagroup "opioids" also contains tapentadol, meperidine, and propoxyphene; Nonopioid medications also includes pregabalin, tricyclic antidepressants, and carisoprodol; Nonopioid recreational substances also includes phencyclidine, MDMA. Time interval change statistics are shown for drug groups with 30 or more nonprescribed positives on UDT 1.

UDT-urine drug test.

Statistically significant trend in the decline of nonprescribed drug group positivity from UDT2-UDT3 $(\mathrm{p}<0.01)^{* * *},(\mathrm{p}<0.05)^{*}$.

the test frequency a patient should receive; instead, frequency should be guided by patient acuity and level of care. For substance misuse patients in early recovery, the ASAM panel noted that as the patient becomes more stable in recovery, drug testing should be performed lest often but should still be done at least monthly. Individual consideration may be given for less frequent testing if a patient is in stable recovery. ${ }^{7}$ Our results suggest that given the high levels of drug misuse in the general drug monitoring population we studied, these guidelines may be applicable to patients receiving chronic opioid 


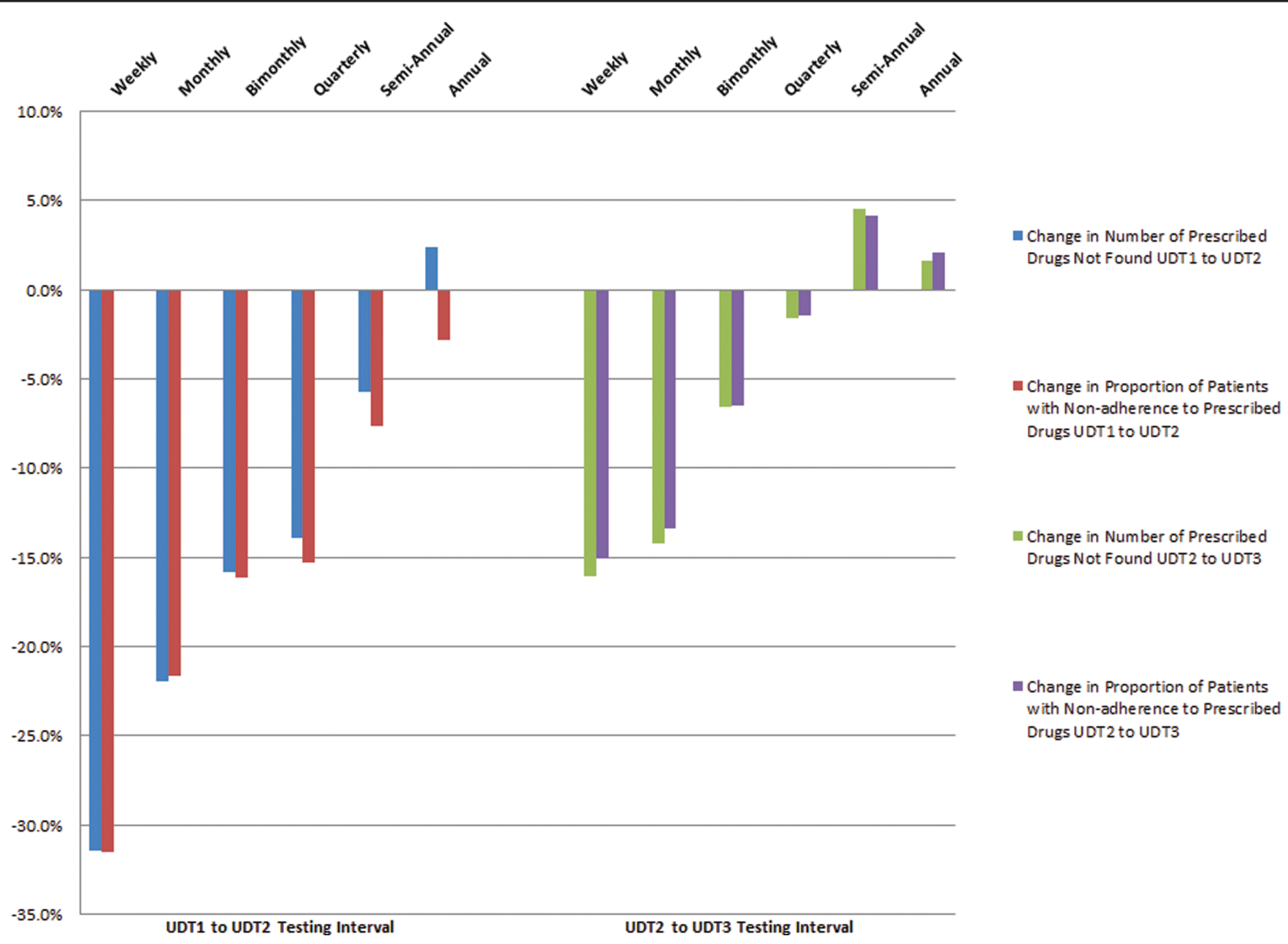

Figure 5. Change in number of noncompliance results and proportion of patients demonstrating noncompliance for prescribed drugs from UDT1 to UDT2 and from UDT2 to UDT3, by testing interval. UDT-urine drug test. Patients can be tested at different time intervals from UDT1-UDT2 and UDT2-UDT3.

therapy as well as those in addiction treatment. However, we also realize that despite the exclusion of patients from SUD facilities, medication-assisted treatment can occur in both primary care and pain management settings and our general drug monitoring population may include SUD patients and weekly testing may disproportionately include these patients.

Heroin and nonprescribed fentanyl are the drugs most responsible for the soaring overdose death rates in the United States. The patterns of positivity demonstrated in this study suggest that clinical strategies including short-interval follow-up UDTs have the potential to reduce misuse of these especially dangerous drug groups. Between UDT1 and UDT2, positivity for heroin and nonprescribed fentanyl declined by roughly one-third among patients tested monthly. However, the populations tested quarterly and semiannually both demonstrated increases in nonprescribed fentanyl positivity (22 percent and 25 percent, respectively). While heroin had the largest overall decline between UDT1 and
UDT2 of any drug group tested (32 percent), it was one of only three drug groups that demonstrated an overall increase in nonprescribed positivity between UDT2 and UDT3 (at 4 percent). It is worth noting that, even between UDT2 and UDT3, the population tested weekly demonstrated a 9 percent decline in heroin positivity. It seems clear that, especially in the case of the most dangerous drugs such as heroin and fentanyl, treatment plans with shorter drug testing intervals were associated with a decline in nonprescribed positivity rates.

Although this study focused primarily on changes in nonprescribed drug use associated with UDTs, changes in noncompliance with prescribed drugs are also very important. There are a number of reasons: patients may not take their prescribed analgesics, including an improvement in their pain, undesirable side effects, or an inability to afford them. Patients may also willingly or unknowingly divert their drugs to others-the so-called "medicine cabinet effect." Lowering rates of noncompliance with prescribed drugs also has the potential to limit diversion, thus 
Table 5. Change in noncompliance with prescribed drug groups from UDT1 to UDT2, by testing interval

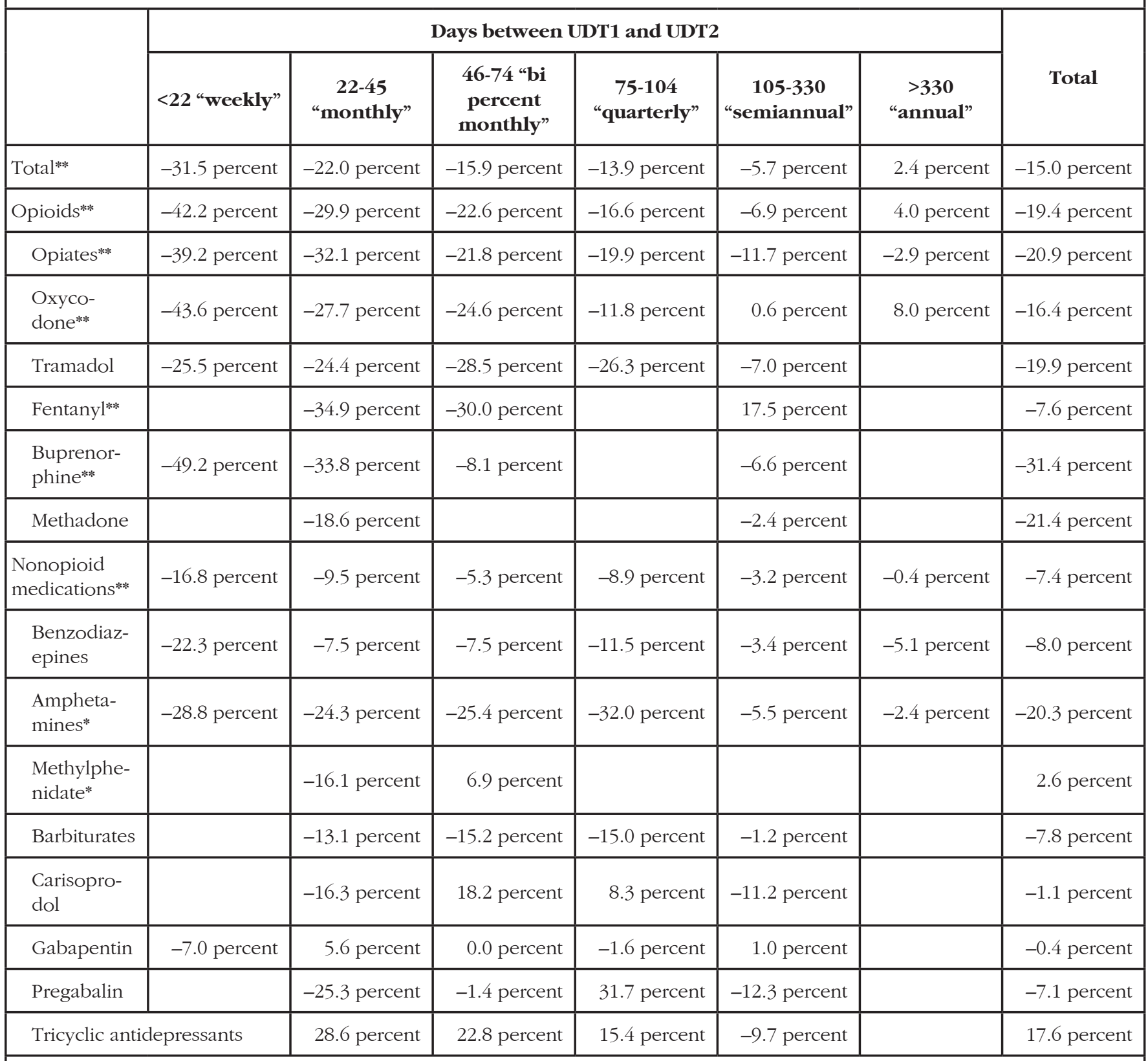

NOTE: The meta-group "opioids" also contains tapentadol, meperidine, and propoxyphene.

UDT—urine drug test.

Time interval change statistics are shown for drug groups with 30 or more noncompliance results on UDT1.

Statistically significant trend in the decline of noncompliance for drug group from UDT1-UDT2 $(\mathrm{p}<0.01)^{* *},(\mathrm{p}<0.05)^{*}$.

lowering the supply of nonprescribed drugs available for potential misuse.

The possible reasons for the effectiveness of treatment strategies that include repeat drug testing are complex. For some patients, the increased accountability for their actions, or fear of possible legal ramifications, may play a large role. Physicians may threaten to stop prescribing particular medications to patients when inappropriate/nonprescribed positive tests occur. It is also possible that physicians simply stop prescribing drugs to patients who are not testing positive for them, leading to less noncompliance. Whatever the reason, declining misuse is important for the patient population in the context 
Table 6. Change in noncompliance with prescribed drug groups from UDT2 to UDT3, by testing interval

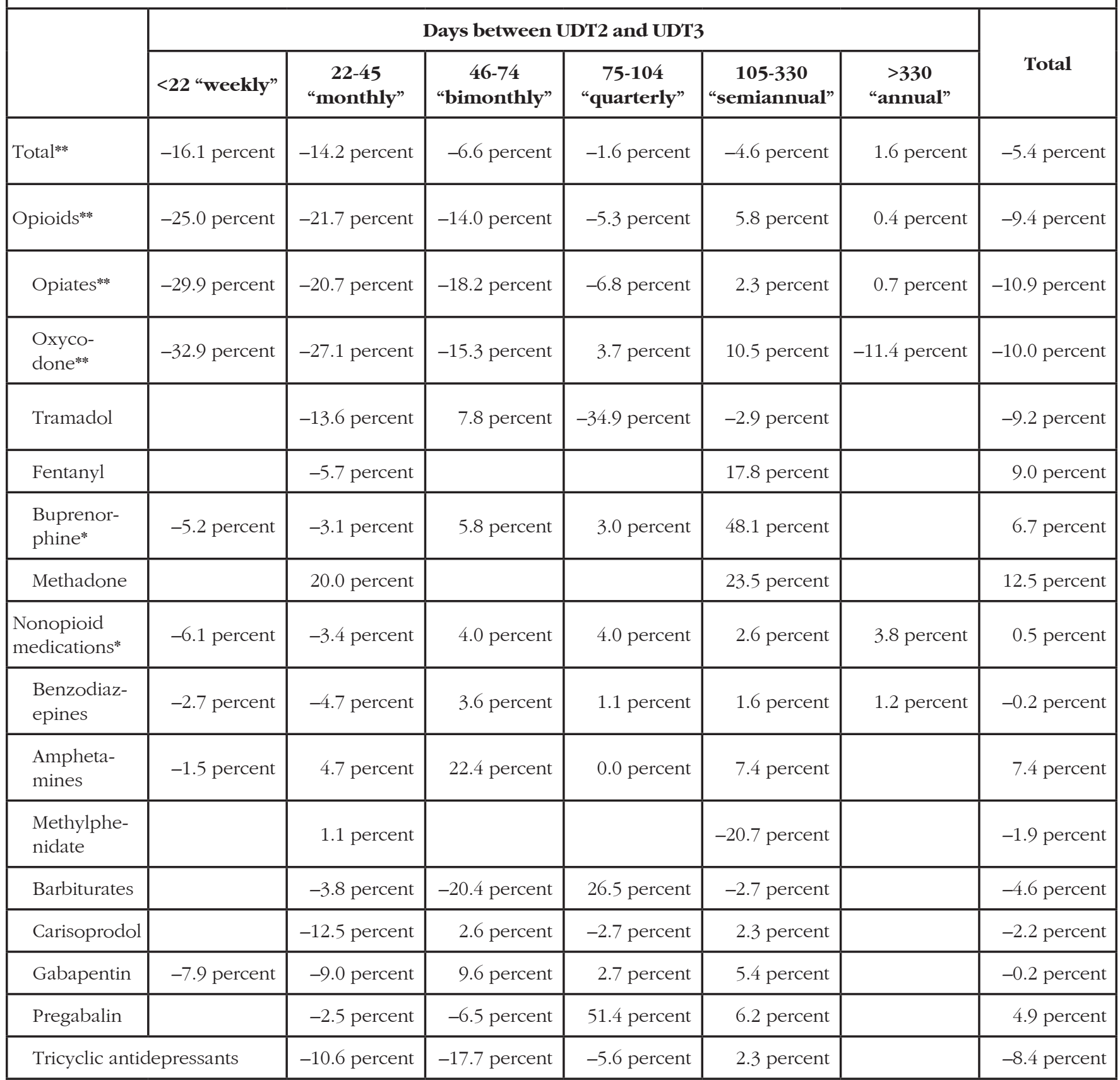

NOTE: The metagroup "opioids" also contains tapentadol, meperidine, and propoxyphene.

UDT-urine drug test.

Time interval change statistics are shown for drug groups with 30 or more noncompliance results on UDT1.

Statistically significant trend in the decline of noncompliance for drug group from UDT2-UDT3 $(\mathrm{p}<0.01)^{* *},(\mathrm{p}<0.05)^{*}$.

of our country's escalating drug overdose and opioid epidemic.

This is the first published study we are aware of that examine changes in prescription drug misuse as the time interval between follow-up testing varies. Although the results of this study may be expected by many clinicians, especially those who frequently drug test their patients, to the best of our knowledge this is the first study that demonstrates changes in aggregate misuse at different time 
intervals between UDTs. Strengths of this study include our large cohort (148,803 UDT results from 49,601 patients) with broad representation of age, sex, physician specialty, and geographic location. Mass spectrometry-obtained drug testing results enabled analysis of demonstrated drug use, as opposed to results obtained through survey results or prescribing databases alone. Including a large number of drug groups in our analysis enabled a more complete picture of drug misuse than a study focused on a single group and allowed for comparison between groups.

We also recognize some study limitations. Drug testing is only part of the larger story of ongoing physician engagement in patient health, and specifically intervention into drug misuse. We have no data on the specific strategies being employed by the physicians treating the patients in this study, or what impact that may have on patient outcomes. We also do not know whether a UDT was prescheduled with patient knowledge or if testing was random. We also had no knowledge of testing at other labs or ongoing treatment by other providers prior to the results from UDT1. Medications can also be prescribed on an "as-needed" basis, thus, in some cases a negative result for a prescribed drug may not constitute misuse. Physician prescribing information may not be perfect, but any mistakes may also reflect lack of physician knowledge about other prescribed drugs, which can have dangerous implications.

We did not analyze patients' medical history, which may affect both which patients were tested and the number of times they were tested. It is also possible that some patients may have had UDTs at other laboratories before testing began at Quest Diagnostics, potentially affecting their behavior. In some cases, a patient may have an agreement in place with a physician that demonstration of nonprescribed drug use will result in the discontinuation of treatment (and therefore testing). In other cases, a physician may see a UDT without misuse, or multiple UDTs without misuse, as a reason to discontinue testing. How the balance of these factors has impacted the evolution and misuse rates of patients included in our cohort is unclear. Despite the CDC recommendations to drug test all patients being prescribed opioids, we understand that in many cases patients who are tested are likely to be considered higher risk by the ordering clinician. Thus, we consider the rates reported in this study reflective of the drug-monitored patient population and not necessarily reflective of all patients receiving controlled substances.

\section{CONCLUSION}

Although prescription monitoring program data reveal what controlled substance prescriptions a patient is filling at the pharmacy, drug testing is the only objective tool available to determine what substances the patient is actually taking. Repeat drug testing was associated with declines in drug misuse for every demographic factor we studied, with the largest declines in misuse being observed for opioid drugs. Shorter time intervals between followup tests were associated with the largest declines in drug misuse. Annual and semiannual drug testing intervals were not associated with consistent declines in nonprescribed drug positivity or compliance with prescribed medications. In addition, weekly and monthly drug testing intervals were associated with consistently larger declines in drug misuse than patients tested bimonthly or quarterly.

We hope that this large-scale data review will stimulate prospective, controlled trials to not only further evaluate how often to drug test patients, but also to help document what specific substances clinicians should be routinely testing for. For now, clinicians who monitor patients for potential drug misuse should consider treatment strategies that include testing more frequently than "at least once annually" (as recommended by the CDC) based on these findings.

Conflicts of interest: Quest Diagnostics provided support in the form of salaries for FLM, JKN, and HWK and consulting fees for JG and speaking fees for $N M$, but did not have any additional role in the study design, collection, analysis, interpretation of data, writing of the manuscript, or decision to publish. None of the authors have any other conflict of interest to report as it relates to this manuscript.

Jeff Gudin, MD, Quest Diagnostics, Secaucus, New Jersey; Rutgers New Jersey Medical School, Newark, New Jersey.

Neel Mehta, MD, Weill Cornell Pain Management, New York-Presbyterian Hospital, New York.

F. Leland McClure, PhD, Quest Diagnostics, Secaucus, New Jersey.

Justin K. Niles, MA, Quest Diagnostics, Secaucus, New Jersey.

Harvey W. Kaufman, MD, Senior Medical Director, Medical Informatics—Quest Diagnostics, Secaucus, New Jersey. 


\section{REFERENCES}

1. Hedegaard H, Miniño AM, Warner M: Drug overdose deaths in the United States, 1999-2017. NCHS Data Brief, no 329. Hyattsville, Maryland: National Center for Health Statistics; 2018. Available at https://www.cdc.gov/nchs/products/databriefs/ db329.btm. Accessed February 12, 2019.

2. Scholl L, Seth P, Kariisa M, et al.: Drug and opioid-involved overdose deaths-United States, 2013-2017. MMWR Morb Mortal Wkly Rep. 2019; 67: 1419-1427.

3. Buchanich JM, Balmert LC, Williams KE, et al.: The effect of incomplete death certificates on estimates of unintentional opioid-related overdose deaths in the United States, 1999-2015. Public Health Rep. 2018; 133: 423-431.

4. Dowell D, Haegerich TM, Chou R: CDC guideline for prescribing opioids for chronic pain-United States, 2016. MMWR Recomm Rep. 2016; 65: 1-49.

5. Gilbert JW, Wheeler GR, Mick GE, et al.: Importance of urine drug testing in the treatment of chronic noncancer pain: Implications of recent medicare policy changes in Kentucky. Pain Phys. 2010; 13(2): 167-186.

6. Standridge JB, Adams SM, Zotos AP: Urine drug screening: A valuable office procedure. Am Fam Phys. 2010; 81(5): 635-640.

7. Consensus Statement. Appropriate use of drug testing in clinical addiction medicine. Available at https://www.asam.org/docs/ default-source/quality-science/appropriate_use_of_drug_test ing_in_clinical-1-(7).pdf?sfursn=2. Accessed September 11, 2018.
8. McCarberg BH: A critical assessment of opioid treatment adherence using urine drug testing in chronic pain management. Postgrad Med 2011; 123(6): 124-131.

9. Katz N, Fanciullo GJ: Role of urine toxicology testing in the management of chronic opioid therapy. Clin J Pain. 2002; 18(4 Suppl): S76-S82.

10. Blum K, Han D, Femino J, et al.: Systematic evaluation of "compliance" to prescribed treatment medications and "abstinence" from psychoactive drug abuse in chemical dependence programs: Data from the comprehensive analysis of reported drugs. PLOS ONE. 2014; 9(9): e104275.

11. Pesce A, West C, Rosenthal M, et al.: Illicit drug use in the pain patient population decreases with continued drug testing. Pain Phys. 2011; 14: 189-193.

12. Manchikanti L, Manchukonda R, Pampati V, et al.: Does random urine drug testing reduce illicit drug use in chronic pain patients receiving opioids? Pain Phys. 2006; 9(2): 123-129.

13. Starrels JL, Becker WC, Alford DP, et al.: Systematic review: Treatment agreements and urine drug testing to reduce opioid misuse in patients with chronic pain. Ann Intern Med. 2010; 152(11): 712-720.

14. Jamison RN, Ross EL, Michna E, et al.: Substance misuse treatment for high-risk chronic pain patients on opioid therapy: A randomized trial. Pain. 2010; 150(3): 390-400.

15. United States Department of Agriculture Economic Research Service: Rural-urban continuum codes. Available at $h t t p: / / w w w$. ers.usda.gov/data-products/rural-urban-continuum-codes/. aspx. Accessed January 10, 2019. 